\title{
El Estado de derecho y la condicionalidad del fondo de recuperación: ¿bloqueo institucional o falta de voluntad política?
}

\section{The Rule of Law and the Recovery Fund's Conditionality: Institutional Deadlock or Lack of Political Will?}

\author{
Guillermo Íñiguez ${ }^{1}$
}

Recibido: 05-09-20

Aceptado: 12-10-20

\section{Resumen}

El gran ausente en las negociaciones del fondo europeo de recuperación volvió a ser el estado de derecho. Este artículo planteará una cuestión de gran importancia constitucional: ¿es posible obligar a los estados miembro a respetar los "valores fundamentales" de la UE? Comenzará detallando tres de los mecanismos contenidos en los tratados: el procedimiento de infracción, el "Rule of Law mechanism" y el artículo 7 TUE. A continuación, desarrollará la tesis, promulgada por Kelemen, del "equilibrio autoritario", que servirá para explicar el comportamiento de las instituciones europeas. Tras analizar diversas propuestas para condicionar el fondo de recuperación, las cuales no presentan dificultades jurídicas, se concluirá que la inacción de las instituciones europeas ante el deterioro democrático en Polonia y Hungría no se debe a una falta de instrumentos legales o de competencias, sino, sobre todo, a una falta de voluntad política.

Palabras-clave: Unión Europea, fondo de recuperación, Estado de derecho, Polonia, Hungría.

\footnotetext{
${ }^{1}$ (g.iniguez-martinez@1se.ac.uk). Graduado en Derecho en la Universidad de Cambridge (Reino Unido). Becario de la Fundación Ramón Areces en la London School of Economics and Political Science. Entre sus publicaciones cabe contar Quo vadis, Consilium? “Agenda Pública”. Recuperado de http://agendapublica.elpais.com/quo-vadis-consilium/ (2020) y El estado de derecho, el gran ausente en el fondo de recuperación, "Agenda Pública". Recuperado de http://agendapublica.elpais. com/el-estado-de-derecho-el-gran-ausente-en-el-fondo-de-recuperacion/ (2020).
} 


\begin{abstract}
The elephant in the room during the recovery fund negotiations was, once again, the rule of law. This paper will address a question of great constitutional importance: is it possible to force Member States to respect the Union's "fundamental values"? It will begin by laying out three of the main mechanisms available to the European institutions: the infringement proceedings, the Rule of Law mechanism and article 7 TEU. It will then develop the thesis, laid down by Kelemen, of the Union's "authoritarian equilibrium", which will serve to explain the European institutions' behaviour. After analysing several proposals for the conditionality of the recovery fund, none of which present major legal obstacles, it will conclude that the European institutions' inaction, faced with democratic backsliding in Hungary and Poland, is not owed to a lack of legal instruments or of competences, but, first and foremost, to a lack of political will.
\end{abstract}

Key-words: European Union, Recovery Fund, Rule of Law, Poland, Hungary.

\title{
1. Introducción
}

El gran ausente en las negociaciones del fondo europeo de recuperación fue, sin duda, el Estado de derecho (Íñiguez, 2020a). Pese a las contundentes declaraciones de Emmanuel Macron, que llegó a afirmar que "sin Estado de derecho, ni un solo euro", el acuerdo ignoró la cuestión, indicando meramente que "un régimen de condicionalidad" sería negociado más adelante. Las conclusiones de la cumbre extraordinaria del Consejo Europeo, que advierten que "[1]os intereses financieros de la Unión se protegerán de conformidad con los principios generales consagrados en los Tratados de la Unión, en particular los valores del artículo 2 del TUE"2 - libertad, democracia, y estado de derecho ${ }^{3}$-, plantean una cuestión de gran importancia constitucional: ¿es posible obligar a los estados miembro a respetar dichos valores?

La regresión democrática sufrida por Europa central a lo largo de la última década ha sido ampliamente documentada, tanto por académicos (Pech y Scheppele, 2017; Müller, 2015; Szante, 2017) como por organismos internacionales ${ }^{4}$. Este artículo explorará la posibilidad, sugerida en el propio

${ }^{2}$ Vid. Unión Europea: Consejo Europeo, Reunión extraordinaria del Consejo Europeo, 17-21 de julio de 2020 - Conclusiones. 21 de julio de 2020. Recuperado de https://www.consilium.europa.eu/ media/45124/210720-euco-final-conclusions-es.pdf

${ }^{3}$ La Unión Europea (UE), según reza el artículo 2 del Tratado de Unión Europea (TUE), "se fundamenta en los valores de respeto de la dignidad humana, libertad, democracia, igualdad, Estado de derecho y respeto de los valores humanos."

${ }^{4}$ Cabe destacar, entre otros, los informes de la Comisión de Venecia (2013), el Parlamento Europeo 
acuerdo alcanzado por el Consejo Europeo el pasado mes de julio ${ }^{5}$, de condicionar los fondos de recuperación al respeto por el estado de derecho. Pese al acertado análisis de Ciannetti et al. (2018), que observan que países como Bulgaria, la República Checa o Malta se encaminan hacia modelos autoritarios similares, este artículo se centrará Polonia y Hungría, cuyos casos permiten analizar el comportamiento de las instituciones europeas a lo largo de los últimos años y sacar conclusiones de cara al futuro.

El artículo comenzará explorando tres de los principales mecanismos, contenidos en los tratados europeos, para velar por el cumplimiento del artículo 2 TUE. A continuación, desarrollará la tesis, promulgada por Kelemen, del "equilibrio autoritario", mediante la cual se analizará el comportamiento de las instituciones europeas. Tras evaluar dos alternativas para condicionar el fondo de recuperación y el Marco financiero plurianual (MFP) 2021-27, concluirá que la ausencia de condicionalidad efectiva no se debe solamente a las deficiencias en el diseño institucional de la Unión Europea, sino que la ausencia de voluntad política, sobre todo por parte de la Comisión Europea, juega un papel notable.

\section{La regresión democrática en Polonia y Hungría}

Los gobiernos de Polonia y Hungría libran, desde hace años, una cruzada contra las instituciones democráticas de sus respectivos países (Íñiguez, 2020a). Dicha tendencia ha quedado reflejada, detalladamente, en el reciente "Rule of Law Report 2020" (Informe sobre el estado de derecho 2020), presentado por la Comisión Europea (2020a) el pasado mes de septiembre. La conclusión es clara: una deriva autoritaria en ambos países, y una Comisión Europea "gravemente preocupada" por sus recientes reformas políticas ${ }^{6}$.

En Polonia, escribe Cliffe, la reelección del presidente Andrzej Duda, tras una campaña caracterizada por su homofobia y su antisemitismo, ha supuesto un duro golpe para la oposición y para unas instituciones cada vez más mermadas ${ }^{7}$ En Hungría, impera desde 2010 un "autoritarismo competitivo" -un régimen híbrido, escriben Levitsky y Way (2019), el cual mantiene una apariencia democrática, pero que incumple los requisitos mínimos para ser considerado como tal (Lendavi, 2017; Levitsky y Way, 2019). El polémico decreto de estado de emergencia, aprobado para afrontar la crisis del coronavirus

(2018), y la Comisión Europea (2020b).

5 Vid. no. 2.

${ }^{6}$ Lluís Pellicer (30 de septiembre, 2020). La UE reabre la batalla con Polonia y Hungría por su deriva autoritaria. El País. Recuperado de https://elpais.com/internacional/2020-09-30/bruselasreabre-la-batalla-con-polonia-y-hungria-por-su-deriva-autoritaria.html.

7 Jeremy Cliffe (15 de julio, 2020). Why second terms for illiberal leaders are different, and more dangerous, than first ones. The New Statesman. Recuperado de https:/www.newstatesman. com/2020/07/why-second-terms-illiberal-leaders-are-different-and-more-dangerous-first-ones 
y que incluía penas de cárcel para periodistas que publicasen "noticias falsas o desestabilizadoras", así como el reciente despido de Szabolcs Dull, editor del medio independiente index. $h u^{9}$, son los últimos ejemplos de sus ataques contra la oposición democrática, denunciado exhaustivamente por la Comisión Europea en el citado informe anual de 2020.

\section{El marco jurídico: la protección del Estado de derecho en los trata- dos europeos}

La Unión Europea (UE), según reza el artículo 2 del Tratado de Unión Europea (TUE), "se fundamenta en los valores de respeto de la dignidad humana, libertad, democracia, igualdad, Estado de derecho y respeto de los valores humanos". El respeto por dichos valores es imprescindible a la hora de acceder a la UE (Kochenov, 2004): los criterios de Copenhague - trazados en el 1993 de cara a la ampliación de 2004, e incorporados a los Tratados mediante el artículo 49 TUE- subrayan la importancia de la "estabilidad de instituciones que garanticen la democracia, el estado de derecho, los derechos humanos, y el respeto por, y la protección de, las minorías"10. Tanto el TFUE como el TUE resaltan su importancia - reiterada por la Carta de Derechos Fundamentales de la Unión Europe- ${ }^{11}$, y proporcionan instrumentos para asegurar su cumplimiento.

Esta sección explorará tres de los principales mecanismos disponibles para las instituciones europeas: el procedimiento de infracción, el "Rule of Law mechanism" (mecanismo para el estado de derecho), y el artículo 7 TUE. Ninguno de los tres, se mostrará, es del todo adecuado para afrontar la regresión democrática en Polonia y Hungría - bien por su rigidez, como en el caso del artículo 7 TUE; por su contexto político, como ha demostrado el Rule of Law Mechanism; o por ambos, en el caso del procedimiento de infracción.

\subsection{La vía judicial: la ineficiencia del procedimiento de infracción}

El principal mecanismo para asegurar el cumplimiento de los tratados por parte de los Estados miembro es el procedimiento de infracción, regulado por

\footnotetext{
${ }^{8}$ K.L. Scheppele (21 de marzo, 2020). Orbán's Emergency. Hungarian Spectrum. Recuperado de https://hungarianspectrum.org/2020/03/21/kim-lane-scheppele-orbans-emergency/?fbclid=IwAR3oT 6sRvSfDpZymerXQtceYbQnMQ-HF8WpoZG0wuhOjuftwvZipMXSVmck

9 Théo Boucart (28 de agosto, 2020). Orbán, the Press, and Europe's Billions. Le Taurillon. Recuperado de https://www.taurillon.org/orban-the-press-and-europe-s-billions

10 Según el artículo 49 TUE, "cualquier Estado europeo que respete los valores mencionados en el artículo 2 y se comprometa a promoverlos podrá solicitar el ingreso como miembro en la Unión." Dichos criterios fueron fijados en el Consejo Europeo de Copenhague en 1993, y desarrollados en el Consejo Europeo de Madrid en 1995.

${ }^{11}$ Cabe destacar los siguientes: Artículo 19 TFUE; Artículos 2, 3, 6 y 7 TUE; y el Artículo 47 de la Carta de Derechos Fundamentales.
} 
los artículos 258-260 TFUE. Según el artículo 258, si la Comisión estima que un Estado miembro ha "incumplido una de las obligaciones que le incumben en virtud de los Tratados", deberá emitir un "dictamen motivado al respecto, después de haber ofrecido a dicho Estado la posibilidad de presentar sus observaciones." En el caso de que este último "no se atuviere a este dictamen", la Comisión podrá recurrir ante el Tribunal de Justicia de la Unión Europea (TJUE).

El procedimiento de infracción fue el mecanismo predilecto de la comisión Juncker para combatir las violaciones sistémicas del estado de derecho por parte de Polonia y Hungría: fue mediante dicho proceso, por ejemplo, que se anuló la reforma judicial polaca ${ }^{12}$, se ilegalizó la ley húngara de transparencia sobre ONG extranjeras (la llamada "ley Stop Soros"13), y se condenó a Polonia, Hungría y la República Checa por negarse a cumplir con el acuerdo de distribución de refugiados alcanzado en $2015^{14}$.

La vía judicial, sin embargo, ha demostrado ser lenta y costosa -según Gormley (2017:65), es el "queso suizo" de los tratados europeos: atractivo a primera vista, con un primer sabor seductor, pero, tras un examen más detenido, poco satisfactorio y lleno de agujeros.

El procedimiento de infracción conlleva una fase administrativa previa, un arduo proceso judicial, y, en última instancia, requiere de la cooperación del Estado miembro demandado. Es aquí, apunta Steinbeis, donde surge el primer obstáculo. La UE, al no ser un Estado en el sentido 'weberiano' - es decir, al carecer del monopolio sobre la fuerza en su territorio - carece del poder coercitivo para asegurarse el acatamiento de las sentencias del TJUE: bastaría la negativa de un Gobierno para abrir una crisis constitucional de difícil resolución ${ }^{15}$. Más allá de este escenario rupturista, la lentitud de los procesos judiciales tiende a vaciarlas de sentido alguno, como ha sido el caso con reciente la sentencia sobre la distribución de refugiados durante la crisis migratoria de 2015. En términos posnerianos ${ }^{16}$, y pese a la reforma introducidas mediante el artículo 260(2) $\mathrm{TUE}^{17}$, el precio a pagar por incumplir los tratados es muy

\footnotetext{
${ }^{12}$ Sentencia del 5 de noviembre de 2019, Comisión/Polonia, C-192/18, ECLI:EU:C:2019:924

${ }_{13}$ Sentencia del 18 de junio de 2020, Comisión/Hungría, C-78/18, ECLI:EU:C:2020:476

${ }^{14}$ Sentencia del 2 de abril de 2020, Comisión/Polonia, Hungría y la República Checa, C-715/17, C-718/17 y C-19/17, ECLI:EU:C:2020:257.

${ }_{15}$ Maximilian Steinbeis (1 de septiembre, 2018). Now let him enforce it. Verfassungsblog. Recuperado de https://verfassungsblog.de/now-let-him-enforce-it/

16 Richard Posner (1981), The Economics of Justice. Cambridge, MA: Harvard University Press.

${ }^{17}$ Según el artículo 260 (2),

"si la Comisión estimare que el Estado miembro afectado no ha adoptado las medidas necesarias para la ejecución de la sentencia del Tribunal ... indicará el importe de la suma a tanto alzado o de la multa coercitiva que deba ser pagada por el Estado miembro afectado y que considere adaptado a las circunstancias. Si el Tribunal declarare que el Estado miembro afectado ha incumplido su sentencia, podrá imponerle el pago de una suma a tanto alzado o de una multa coercitiva".

Vid. Brian Jack (2012). Article 260(2) TFEU: An Effective Judicial Procedure for the Enforcement of Judgements? 19(3) European Law Journal, 404-421.
} 
bajo, lo cual incentiva $-\mathrm{y}$ facilita- el acatamiento selectivo de las sentencias del TJUE.

Las restricciones procesales, a su vez, han limitado la efectividad del procedimiento de infracción. Según el artículo 258, la prerrogativa de iniciar dicho proceso corresponde exclusivamente a la Comisión Europea, la cual decide -sin que su motivación pueda ser recurrida ante, o analizada por, el $\mathrm{TJUE}^{18}$ - cuando iniciar la fase administrativa, así como qué casos llevar ante el TJUE una vez concluida dicha fase. Incluso la aplicación del artículo 259, el cual permite a "cualquier estado miembro" recurrir al TJUE "si estimare que otro Estado miembro ha incumplido una de las obligaciones que le incumben en virtud de los Tratados", requiere una valoración previa por parte de la Comisión, la cual puede emitir un "dictamen motivado" sobre el caso.

Esta llamativa falta de competencias por parte de otras instituciones, como el Parlamento Europeo o el Defensor del Pueblo Europeo, ha sido complementada por una interpretación judicial extremadamente restrictiva, negando derechos procesales a actores privados y dificultando cualquier control administrativo y judicial sobre el ejercicio de dicho mecanismo por parte de la Comisión ${ }^{19}$. Las críticas por parte del Defensor del Pueblo, el cual ha denunciado, en numerosas ocasiones, la falta de transparencia y rendición de cuentas a la hora de aplicar uno de los principales mecanismos judiciales de la $\mathrm{UE}^{20}$, han surtido poco efecto. Más allá de una tímida respuesta por parte de la Comisión - la publicación de un informe detallando procedimientos internos a la hora de aplicar el artículo $258^{21}$-, ha habido poco cambio sustancial. La Comisión, en otras palabras, ha mantenido su discrecionalidad, imponiendo su concepción restrictiva del procedimiento, y por tanto limitando su utilidad.

En su estudio sobre el papel de la Comisión en la reciente involución democrática en Polonia y Hungría. Carlos Closa (2019: 705) descarta que su composición partidista de la Comisión Europea explique su lentitud a la hora de actuar contra Polonia y Hungría. Pese a que los tres últimos presidentes (Barroso, Juncker y von der Leyen) pertenecieron al Partido Popular Europeo, los tres principales partidos europeos (el popular, el socialdemócrata y el liberal) han ocupado carteras relevantes a lo largo de la última década, y no existen pruebas concluyentes, concluye, que muestren una relación directa entre una mayoría popular y una mayor reticencia a la hora de implementar el Rule of Law Framework o activar el artículo 7.

18 Asunto 416/85, Comisión/Reino Unido [1988] ECR 3127.

19 Asunto 48/65, Alfons Lüttike GmbH/Commission [1966] ECLI:EU:C:1966:8, [27]

20 Véase, por ejemplo, las críticas en: Defensor del Pueblo Europeo (2005). Informe especial del Defensor del Pueblo Europeo al Parlamento Europeo a raíz del proyecto de recomendación a la Comisión Europea sobre la reclamación 3453/2005/GG

${ }^{21}$ Comision Europea (2012). Updating the handling of relations with the complainant in respect of the application of Union law. COM (2012) 154 final. 
Es innegable, sin embargo, que el diseño del procedimiento de infracción lo ha sometido a la coyuntura política de cada Colegio de Comisarios, facilitando, de este modo, las represalias por parte de los países infractores. La proactividad de la comisión Juncker a la hora de aplicar dicho instrumento, por ejemplo, tuvo un coste extremadamente elevado: el que fuera el encargado de frenar la reforma judicial polaca de 2015, el entonces vicepresidente primero socialdemócrata Frans Timmermans, vio mermadas sus aspiraciones a presidir la Comisión en 2019 -vetado, precisamente, por los gobiernos de Polonia y Hungría, los cuales declararon que "vetarían" a aquellos que "no les respetasen"22. Ello, sin duda, ayuda a explicar la actitud de Ursula von der Leyen, la cual, como se explicará más abajo, se ha mostrado mucho más reacia a la hora de intervenir.

La utilidad del procedimiento de infracción a la hora de velar por el cumplimiento de los tratados está fuera de toda duda. Su conceptualización, sin embargo, diseñada para responder a violaciones puntuales de directivas o reglamentos, lo hace ineficaz a la hora de combatir violaciones sistémicas del estado de derecho (Gormley, 2017). Es necesario repensar su funcionamiento, otorgando una mayor capacidad de acción al Parlamento Europeo o al Defensor del Pueblo y sometiendo las decisiones de la Comisión a una mayor transparencia. Mientras tanto, la estrategia judicial por si sola es inviable.

\subsection{El "Rule of Law mechanism"}

En 2014, la Comisión Europea presentó su denominado "Rule of Law mechanism", un instrumento creado para llenar el supuesto vacío entre el procedimiento de infracción y el artículo 7 TUE. Mediante dicho instrumento, la Comisión -junto con el Consejo de Europa y la Agencia de los Derechos Fundamentales de la UE- identificaría amenazas "sistémicas" al estado de derecho en los Estados miembro, dialogaría con las sus instituciones nacionales y recomendaría "medidas concretas" para combatir las amenazas en cuestión, evitando, por ello, el uso del artículo 7 TUE $^{23}$.

Sin embargo, lamentan Pech y Scheppele (2017), dicho mecanismo ha sido otra víctima de la falta de ambición de las instituciones comunitarias. El propio planteamiento, añaden, denota un error estratégico: frente a las constantes amenazas y desplantes por parte del gobierno húngaro ${ }^{24}$, la Comisión actuó

${ }^{22}$ PolandIn (24 de junio, 2019). Polish MFA: Central Europe will veto those who don't respect us. PolandIn. Recuperado de https://polandin.com/43207200/polish-mfa-central-europe-will-veto-thosewho-dont-respect-us

${ }^{23}$ European Commission (2014). Communication from the Commission to the European Parliament and the Council. A new EU Framework to Strengthen the Rule of Law. COM(2014) 158 final

${ }^{24}$ Respondiendo al Informe Tavares, aprobado por el Parlamento Europeo en 2013, el gobierno húngaro denunció una "violación" de su soberanía nacional, "rechazando" una Europa "dictatorial" y 
con timidez, planteando el dialogo entre instituciones como la mejor forma de combatir la deriva autoritaria de Budapest y Varsovia (Halmai, 2018: 4-6). Ante una realidad innegable, y una situación que -ya en 2013-presentaba un riesgo existencial para la propia UE, se optó por un instrumento basado en los valores liberales del diálogo, confiando en la cooperación por parte de los propios gobiernos infractores ${ }^{25}$. Ello no solamente no surtió efecto: como denuncian Pech y Scheppele (2017:5), demoró -y por ello dificultó aún más- la aplicación del artículo 7.

Cuando el uso del artículo 7 se puso sobre la mesa, concluyen Pech y Scheppele, la oportunidad de actuar se había perdido: Polonia y Hungría ya habían formado su alianza, y el mecanismo en sí, estigmatizado y relegado a una opción nuclear que debía ser evitada a toda costa, había quedado deslegitimado. Es precisamente este fracaso, tanto estratégico como institucional, el que ha llevado a académicos como Gábor Halmai (2018:1) a concluir que hacen falta "nuevos métodos de condicionalidad" para hacer respetar el artículo 2 TEU, incluido uno que condicione el acceso a los fondos del MPF a su cumplimiento.

\subsection{El artículo 7 TUE y la pasividad del Consejo Europeo}

El artículo 7 del TUE, el cual permite sancionar a los estados por violaciones "graves y persistentes" de los valores fundamentales de la Unión y puede conllevar la pérdida del derecho de voto en el Consejo Europeo, consta de dos partes: un mecanismo preventivo y otro sancionador. En caso de un "riesgo claro de violación grave" de los valores fundacionales por parte de un Estado miembro (artículo 7(1)), a propuesta del Parlamento o de la Comisión y con el apoyo de cuatro quintas partes de los Estados miembro, se activa un "mecanismo preventivo", mediante el cual las instituciones comunitarias evalúan la situación para detectar un posible retroceso democrático. Si dicha violación se vuelve "grave y persistente" (artículo 7(2)), y tras invitar al estado acusado a "presentar sus observaciones" el Consejo Europeo puede, por unanimidad-excluyendo al país acusado-, activar el mecanismo sancionador, pudiendo suspender "determinados derechos derivados de la aplicación de los Tratados al estado miembro", incluido su derecho al voto en el Consejo (artículo 7(3)).

Este instrumento, sin embargo, ha demostrado en repetidas ocasiones ser ineficiente: al requerir el artículo 7(2) la unanimidad del Consejo Europeo, ha

acusando a las instituciones europeas de incumplir el TUE (Halmai, 2018:4).

25 Como subraya Steinbeis (2020), "si hay un problema que la UE no tiene en relación con Hungría [o Polonia o Bulgaria]", ese problema es comprender su deterioro democrático: "cualquiera que esté interesado en saber lo que está ocurriendo", concluye, "lo sabe, y lo lleva sabiendo desde hace mucho tiempo". Vid. Maximilian Steinbeis (2 de octubre, 2020). Quo usque tandem, Viktorina. Verfassungsblog. Recuperado de https://verfassungsblog.de/quo-usque-tandem-viktorina/ 
bastado una alianza abierta entre el nacional-catolicismo polaco y el iliberalismo húngaro para imposibilitar su aplicación y vaciarlo de sentido alguno. Los casos de Polonia y Hungría, de hecho, ilustran la paradoja que rodea al Estado de derecho europeo: dos gobiernos que incumplirían los criterios de Copenhague están impidiendo a la UE defender su propio ordenamiento jurídico, dejando desprotegidos, como ha denunciado en repetidas ocasiones el Parlamento Europeo $^{26}$, a casi 50 millones de ciudadanos europeos (Íñiguez, 2020a).

Es innegable que gran parte del fracaso del artículo 7 se debe a su manipulación por parte de Hungría y Polonia, que han aprovechado el talón de Aquiles de la UE -el requerimiento de unanimidad en las votaciones del Consejo- para impedir su uso. Sin embargo, apunta Scheppele, el veto de un país no tendría por qué significar el fin del proceso. Mientras que el mecanismo sancionador (artículos 7(2)-(3)) requiere el apoyo unánime del Consejo, dicho mecanismo solo puede someterse a votación previa activación del mecanismo preventivo (art 7(1)), que solo requiere el apoyo de 22 gobiernos. Si, en otras palabras, la Comisión, el Parlamento o cuatro quintas partes del Consejo hubieran activado el artículo 7(1) contra Polonia o Hungría a la vez, concluye Scheppele, la aplicación del artículo 7(3) hubiera sido una amenaza real ${ }^{27}$.

El análisis de Scheppele refleja dos de las tendencias que se empiezan a detectar a lo largo de este artículo: la lentitud de las instituciones europeas a la hora de actuar, y el importante papel que han jugado -y que siguen jugandolas alianzas políticas en la respuesta al deterioro democrático en Polonia y Hungría.

\section{4. ¿Un "equilibrio autoritario” en el seno de la Unión?}

\subsection{La teoría del "equilibrio autoritario"}

La ineficacia por parte de la Comisión y del Consejo a la hora de emplear los instrumentos disponibles -el procedimiento de infracción, el "Rule of Law mechanism", y el artículo 7 TUE- ha resultado en lo que Kelemen denomina un "equilibrio autoritario". Pese a su aparente compromiso con los valores democráticos -ilustrado, por ejemplo, por los criterios de Copenhague, o por la introducción del propio artículo 7 a través del Tratado de Niza-, y a cambio de asegurarse la unanimidad en el Consejo, la UE se ha mostrado dispuesta

\footnotetext{
${ }^{26}$ Parlamento Europeo (9 de enero, 2020). Hungría y Polonia: avances en los procedimientos del Artículo 7. Recuperado de https://www.europarl.europa.eu/news/es/agenda/briefing/2020-01-13/5/ hungria-y-polonia-avances-en-los-procedimientos-del-articulo-7.

${ }^{27}$ Kim Lane Scheppele (1 de noviembre, 2016). EU can still block Hungary's veto on Polish sanctions. POLITICO. Recuperado de https://www.politico.eu/article/eu-can-still-block-hungarysorban-veto-on-polish-pis-sanctions/.
} 
a pasar por alto violaciones sistemáticas del Estado de derecho por parte de ciertos gobiernos.

Dicho acuerdo tácito constituye un "equilibrio", siempre según Kelemen, porque aparenta beneficiar a ambas partes. Por un lado, la UE se asegura su funcionamiento: la complejidad de las negociaciones en el Consejo Europeo le obliga, de cara a la galería, a priorizar las crisis más inmediatas -la Covid-19, la recesión económica, la crisis de los refugiados-, relegando así el Estado de derecho, percibido como una cuestión menos urgente, a un segundo plano. Bajo este pretexto se evita el bloqueo institucional que supondría un permanente veto por parte del grupo de Visegrado, imposibilitando la capacidad de reacción de la UE.

Por otro, dichos gobiernos pueden proseguir su cruzada contra las instituciones democráticas de sus respectivos países, con la tranquilidad de saber que no serán sancionados por unas instituciones comunitarias cada vez más dependientes de ellos. La debilidad institucional de una Unión Europa confederal (Rodríguez Aguilera de Prat, 2020), en otras palabras, impide la aplicación correcta de sus propios tratados.

Tres factores sustentan el "equilibrio" denunciado por Kelemen: el sistema de familias políticas europeas, el cual protege a líderes autoritarios; los distintos fondos europeos, que dichos gobiernos han utilizado para mantenerse en el poder y para ampliar sus redes de clientela; y la libertad de circulación, la cual ha promovido la emigración de ciudadanos insatisfechos, debilitando el papel de las distintas oposiciones en la política nacional (Kelemen 2020b:481).

De estos tres, dos son especialmente relevantes para analizar la condicionalidad de los fondos: si la ineficacia de las instituciones europeas se debe exclusivamente a consideraciones partidistas y a alianzas políticas dentro del Consejo (el primer factor que denuncia Kelemen), ningún régimen de condicionalidad será más que una utopía, facilitando aún más el avance de los gobiernos autoritarios de Varsovia y Budapest. Si dicha resistencia -como expone este artículo- se pudiese vencer, un marco legal que dificultase el acceso a fondos europeos, y que por lo tanto mermase la capacidad los gobiernos autoritarios de sustentarse en el poder (el segundo elemento del modelo de Kelemen), podría romper dicho "equilibrio autoritario", inclinando la balanza a favor de las instituciones europeas.

\subsection{El "equilibrio autoritario" en la práctica}

Las negociaciones del fondo de recuperación en julio de 2020 supusieron el enésimo ejemplo del funcionamiento práctico del modelo de Kelemen. Los graves problemas económicos de los estados del sur, así como la necesidad de mantener la confianza de los mercados y de recuperar la imagen de la UE tras su 
tímida respuesta inicial a la pandemia, pesaron más que cuestiones relacionadas con la protección de la democracia. Así, pese a la insistencia de Emmanuel Macron y el cruce de reproches entre Mark Rutte -que planteó el Estado de derecho como línea roja-y Viktor Orbán - que le acusó de "odiar" a Hungría ${ }^{28}$-, el resultado fue un acuerdo de minimis que permitió a ambas partes salvar la cara $^{29}$, pero que al ignorar la condicionalidad del fondo de recuperación parece haber beneficiado a unos gobiernos autoritarios conscientes de su victoria.

Fue precisamente el miedo - razonable- ante un posible desacuerdo lo que permitió a Hungría y Polonia sumar, indirectamente, más estados a su causa: el primer ministro portugués Antonio Costa, temiendo un nuevo bloqueo institucional que retrasase su entrada en vigor, abogó por acordar el fondo de recuperación en un primer lugar, y negociar un régimen de condicionalidad más adelante. Las "cuestiones de valores", añadió Costa tras la cumbre, no debían "cuantificarse", y han de ser resueltas mediante la aplicación del artículo 7 TUE, no mediante la condicionalidad del fondo de recuperación ${ }^{30}$.

Según Closa (2019:699), el conservadurismo por parte de las instituciones europeas no es más que un ejercicio de realpolitik por parte de una UE consciente de que el cumplimiento de los tratados depende, en última instancia, de los gobiernos nacionales. Al concluir que no existe una mayoría que el Consejo Europeo emplee el artículo 7 TUE, por ejemplo, la Comisión, consciente de los riesgos que conllevaría su fracaso - la deslegitimación del propio mecanismo, así como un posible repunte de euroescepticismo en el país en cuestión-, prefiere incentivar el diálogo interinstitucional, con la esperanza de que el gobierno infractor recapacite.

Un modelo que otorga tan poca importancia a la política partidista en el Consejo, la Comisión y el Parlamento, sin embargo, resulta poco satisfactorio.

Por una parte, el sistema de familias políticas, uno de los factores más importantes a la hora de predecir el posicionamiento político de los partidos nacionales (Hooghe, Marks y Wilson, 2002:966), ha permitido a aliados políticos protegerse entre sí sin sufrir coste electoral alguno (Kelemen, 2020:484). La ausencia de listas electorales transnacionales ha facilitado este comportamiento, reduciendo el margen de rendición de cuentas al cual se someten los partidos nacionales, y convirtiendo las familias en "escudos

${ }^{28}$ Lili Bayer y Hans von der Burchard (20 de julio, 2020). Viktor Orbán: I don’t know why Mark Rutte hates me and Hungary. POLITICO. Recuperado de https:/www.politico.eu/article/viktororban-i-dont-know-why-mark-rutte-hates-me-and-hungary/

${ }^{29}$ Bernd Riegert (23 de julio, 2020). La UE condiciona ayudas pospandemia al respeto del Estado de derecho. Deutsche Welle. Recuperado de https://www.dw.com/es/la-ue-condiciona-ayudaspospandemia-al-respeto-del-estado-de-derecho/a-54270629

${ }^{30}$ Joao Barros (13 de julio, 2020). Costa defends an approach in Hungary that separates recovery funds from the rule of law problem. O Jornal Económico. Recuperado de https://jornaleconomico. sapo.pt/en/news/Costa-defends-an-approach-in-Hungary-that-separates-recovery-funds-from-therule-of-law-problem613352\#.Xw7YCFalHvY.twitter 
protectores" hacia estos últimos ${ }^{31}$. Al no existir, por ejemplo, un Partido Popular Europeo que se presente a las elecciones europeas como tal, el coste electoral que dicha marca puede sufrir si uno de sus partidos nacionales (por ejemplo, la CDU alemana) apoya un régimen autoritario en otro país (por ejemplo, el instaurado por Orbán en Hungría) se reduce significativamente.

Esto es precisamente lo que ha ocurrido en el caso de Fidesz. Uno de los artífices de que el partido de Orbán siga perteneciendo al EPP, mantiene Kelemen (2020b:488), es la CDU de Merkel, Ursula von der Leyen o Manfred Weber, con la cual Fidesz mantiene una estrecha relación desde hace años. En 2019, por ejemplo, ante la amenaza de su expulsión del EPP, el secretario general de la CDU, Paul Ziemiak, abogó por una "solución de compromiso" para mantener a los húngaros en su partido ${ }^{32}$. Igualmente, cuando, en enero de 2020, el Parlamento Europeo aprobó una resolución exigiendo mayores avances en la aplicación del artículo 7 contra Hungría y Polonia, varios miembros del EPP -incluido el Partido Popular español- se opusieron ${ }^{33}$.

Por otra parte, los estados autoritarios han tejido alianzas y grupos de presión dentro del propio Consejo Europeo, creando importantes redes de apoyo mutuo como el grupo de Visegrado (Garai 2018). Un mecanismo que amenace con sancionar violaciones del estado de derecho en Hungría o Polonia, por lo tanto, será vetado no solo por dichos países, sino por una serie de aliados, como Bulgaria, Malta o la República Checa, que entienden que ese mismo instrumento podrá ser usado contra ellos en el futuro. Esta resistencia frente a lo que es visto como una injerencia por parte de las instituciones comunitarias ha generado, por tanto, una "minoría de bloqueo", capaz de ralentizar el funcionamiento de un organismo confederal (Rodríguez Aguilera de Prat, 2020).

El primer año de la "comisión von der Leyen" parece corroborar la tesis de Kelemen. A lo largo de su primer año de mandato, la estrategia de von der Leyen ha consistido en tender la mano y evitar confrontaciones con Varsovia y Budapest, dos gobiernos que resultaron decisivos para su elección, tanto en el Consejo como en el Parlamento: en unas polémicas declaraciones en 2019, aseguró que "nadie es perfecto", y manifestó su convicción de que todos los estados miembros "aspiran" a un Estado de derecho ${ }^{34}$. El polémico Pacto Sobre Migraciones y Asilo, el cual renuncia a las cuotas obligatorias y facilita

${ }^{31}$ Bernardo de Miguel y Lluis Pellicer (21 de marzo, 2019). La derecha europea elude expulsar a Orbán y opta por una suspensión. El País. Recuperado de https:/elpais.com/internacional/2019/03/20/ actualidad/1553092068 648405.html; E. Zgut (11 de junio, 2019). Orbán’s Cat and Mouse Game. Visegrad Insight. Recuperado de https://visegradinsight.eu/orbans-cat-and-mouse-game/

${ }^{32}$ Hungary Matters (3 de julio, 2020). Hungary govt officials hold talks in Berlin. Recuperado de http://hungarymatters.hu/2020/07/03/hungary-govt-officials-hold-talks-in-berlin/

33 Bernardo de Miguel (16 de enero, 2020). El PP se desmarca de la presión del Parlamento Europeo para preservar la independencia judicial en Polonia. El País. Recuperado de https://elpais. com/politica/2020/01/16/actualidad/1579186079_451289.html

34 Andrew Rettman (19 de julio, 2020). Von der Leyen signals soft touch on migrants, rule of law. Recuperado de https://euobserver.com/news/145504. 
la expulsión de inmigrantes irregulares por parte de los estados miembro ${ }^{35}$, parece confirmar esta tendencia: el grupo de Visegrado, un grupo de presión creado para facilitar la entrada de sus miembros en la UE, ejerce una influencia cada vez mayor, tanto sobre las decisiones del Consejo Europeo como sobre las políticas de la Comisión ${ }^{36}$.

La ambigüedad que subyace a la política de von der Leyen quedó bien ejemplificada en su reciente discurso sobre el estado de la Unión Europea, pronunciado ante el Parlamento Europeo el pasado mes de septiembre. Dicha intervención incluyó cinco referencias al Estado de derecho, así como una clara alusión a la discriminación LGTBI en Polonia. Sin embargo, pese a su declaración de que los fondos transferidos a los Estados miembros serán protegidos "contra todo tipo de fraude, corrupción y conflicto de intereses", así como su promesa de que la Comisión "no tolerará" violaciones del Estado de derecho, von der Leyen omitió cualquier referencia su posible condicionalidad ${ }^{37}$.

Su estrategia, por lo tanto, parece clara: intentar, en la medida de lo posible, ofrecer la zanahoria y esconder el palo, pese a lo evidente que resulta que la situación en Polonia y Hungría -y, en menor medida, en la República Checa o en Malta- no tiende hacia más democracia, sino hacia un autoritarismo cada vez más descarado.

Por ello, la deficiente arquitectura institucional de los Tratados - estudiada minuciosamente por Closa, y que pueden servir para explicar la lentitud por parte de las instituciones europeas- no puede ocultar la innegable falta de voluntad política mostrada por miembros de la Comisión y del Consejo. Una vez analizado el papel de las familias políticas en el comportamiento de las tres instituciones, la conclusión de Kelemen -que el presente "equilibrio" se sostiene, en gran medida, porque beneficia a ambas partes- resulta difícil de ignorar.

${ }^{35}$ Gonzalo Fanjul y Ana González-Páramo (28 de septiembre, 2020). Europa se coloca en el lado equivocado de la historia. Política Exterior. Recuperado de https://www.politicaexterior.com/europase-coloca-en-el-lado-equivocado-de-la-historia/

36 Según apunta Sierakowski (2020), otro factor que puede explicar la estrategia de la Comisión es un cálculo geopolítico: antagonizar al grupo de Visegrado -y, por lo tanto, acercar a sus miembros a la órbita rusa-, perjudicaría tanto a Europa como a Alemania, que mantiene una importante relación comercial con Hungría, Polonia y la República Checa - comparable a aquella con Francia, China o EEUU (Statistisches Bundesamt, 2020).

37 Comisión Europea (16 de septiembre, 2020). State of the Union Address by President von der Leyen at the European Parliament Plenary. Recuperado de https://ec.europa.eu/commission/ presscorner/detail/en/SPEECH_20_1655 


\section{La condicionalidad de los fondos europeos: ¿utopía o falta de voluntad política?}

\subsection{El marco plurianual financiero y el fondo de recuperación}

La respuesta del Parlamento Europeo tras la cumbre del Consejo Europeo, en la cual se aprobaron tanto el fondo de recuperación como el MPF 2021-27, no se ha hecho esperar: en una carta dirigida a Angela Merkel, en su calidad de presidenta rotatoria del Consejo Europeo, y a Ursula von der Leyen, los cuatro principales grupos parlamentarios han anunciado que sólo apoyarán un paquete que "realmente" obligue a respetar los valores democráticos. La solución, añaden, pasa por una condicionalidad más estricta, una mayor capacidad para bloquear fondos y un mecanismo que proteja a los destinatarios de dichos fondos, impidiendo apropiaciones indebidas por parte de los gobiernos ${ }^{38}$.

En el caso de Hungría y Polonia, supeditar el acceso a fondos europeos al cumplimiento del artículo 2 del TUE podría ser especialmente eficaz: Polonia fue el país más beneficiado por los fondos estructurales 2014-2020, mientras que Hungría, según escribe Christian Keszthelyi en el 'Budapest Business Journal', depende de la UE para financiar el 95\% de sus inversiones públicas. Dificultar el acceso de Hungría al MFP 2021-27 tendría consecuencias muy visibles sobre su economía: si bien su PIB creció un 4.6\% entre 2006 y 2015, añade Keszthelyi, la imposibilidad de acceder a los fondos europeos durante el ciclo 2007-2013 hubiera reducido dicha cifra a un 1.8\%. Asimismo, el consumo, el cual creció un 5.3\% en dicho periodo, hubiera caído un $11 \% \sin$ la ayuda de la $\mathrm{UE}^{39}$.

¿Sería concebible cortar el grifo a los países que atenten contra el Estado de derecho? Según apuntan Kelemen y Scheppele (2018), los tratados ya contemplan dicha posibilidad ${ }^{40}$. El Reglamento de Disposiciones Comunes, el cual regula la aplicación de los fondos estructurales y de inversión europeos (Fondos EIE), permite la suspensión "total o parcial" de los pagos de dichos fondos cuando exista "una deficiencia grave en el funcionamiento efectivo del sistema de gestión y control del programa operativo"41. Sin Estado de

38 Vlagyiszlav Makszimov (26 de agosto, 2020). Parliament demands rule of law mechanism before signing off EU Budget. Euractiv. Recuperado de https://www.euractiv.com/section/justicehome-affairs/news/parliament-demands-rule-of-law-mechanism-before-signing-off-on-eu-budget/

${ }^{39}$ Christian Keszthelyi (30 de marzo, 2017). Hungary's economy heavily depends on EU funds, study finds. Budapest Business Journal. Recuperado de https://bbj.hu/economy/hungarys-economyheavily-depends-on-eu-funds-study-finds_130880

${ }^{40}$ Kim Lane Scheppele y Daniel Kèlemen (10 de septiembre, 2018). How to Stop Funding Autocracy in the EU. Recuperado de https:/verfassungsblog.de/how-to-stop-funding-autocracy-inthe-eu/

${ }^{41}$ Art. 142(a) del Reglamento (UE) n 1303/2013 del Parlamento Europeo y del Consejo, de 17 de diciembre de 2013, por el que se establecen disposiciones comunes relativas al Fondo Europeo de Desarrollo Regional, al Fondo Social Europeo, al Fondo de Cohesión, al Fondo Europeo Agrícola de 
derecho y una administración judicial independiente, añaden los autores, el "funcionamiento efectivo" de su gestión resulta impensable ${ }^{42}$.

Partiendo de un análisis similar, Israel Butler, director de incidencia política de Civil Liberties Union for Europe, propone un modelo más detallado. El nuevo Marco Financiero Plurianual, señala, "proporciona una oportunidad para que los gobiernos e instituciones de la UE creen nuevas formas de proteger los valores europeos basándose en el poder económico de la Unión" (Butler, 2018a:2). Este fin se podría llevar a cabo, sostiene, mediante un modelo "que permita a la UE suspender los [Fondos EIE] cuando un Estado miembro viole el Estado de derecho".

La propuesta de Butler supondría incorporar, a la regulación del MPF y del fondo de recuperación, la jurisprudencia del TJUE, mencionada anteriormente, sobre la importancia de la independencia judicial en la ejecución de los Fondos EIE. Dicho régimen contemplaría dos posibilidades. En caso de que la Comisión Europea apreciase una "deficiencia grave" en el sistema de gestión de los fondos, podría proceder a su suspensión, condicionando su reactivación a una serie de medidas. Cuando, por otra parte, existiese el riesgo de que dicha "suspensión” tuviera "consecuencias graves y negativas para la población” del país en cuestión, la Comisión podría "asumir la administración de los mismos y quitarle (SIC) esa competencia a las autoridades nacionales" (Butler, 2018a:3) ${ }^{43}$.

El modelo de condicionalidad propuesto por Butler no debería presentar, en sí, problemas jurídicos. La dificultad política, sin embargo, es mayúscula. Al tratarse del MFP, debe aprobarse mediante el proceso legislativo especial - es decir, con el apoyo unánime del Consejo (art 312(1) TFUE). Pese al papel fundamental del Parlamento Europeo, que debe aprobarlo por mayoría absoluta (art 312(2)), y pese a la disconformidad mostrada por los principales grupos parlamentarios con el acuerdo alcanzado por el Consejo, resulta difícil imaginar un contexto político en el cual el Parlamento tumbase el frágil acuerdo alcanzado en julio, obligando al Consejo Europeo a renegociar a la baja.

Desarrollo Rural y al Fondo Europeo Marítimo y de la Pesca, y por el que se establecen disposiciones generales relativas al Fondo Europeo de Desarrollo Regional, al Fondo Social Europeo, al Fondo de Cohesión y al Fondo Europeo Marítimo y de la Pesca, y se deroga el Reglamento (CE) n ${ }^{\circ} 1083 / 2006$ del Consejo.

42 Kelemen y Scheppele también apuntan al artículo 74(3) del reglamento, según el cual "los Estados miembros garantizarán que se pongan en práctica medidas eficaces para el examen de las reclamaciones relacionadas con los Fondos". La importancia de que este sistema respete el artículo 47 de la Carta de Derechos Fundamentales, así como con el artículo 19 TUE, ha sido subrayada por el TJUE en Liivimaa Lihaveis MTÜ (Asunto 562/12).

${ }^{43}$ La propuesta de Butler, por lo tanto, permitiría vencer una de las principales objeciones al régimen de condicionalidad: que las víctimas no serían los gobiernos autoritarios, sino sus ciudadanos, lo cual podría resultar en un mayor escepticismo en dichos países (vid. Halmai (2018:14); Manuel Müller (31 de mayo, 2017). Geld nur bei Wohlverhalten: Sind politische Bedingungen der EU-Strukturfonds die Lösung für Ungarn und Polen? Der (europäische) Föderalist. Recuperado de https://www.foederalist. eu/2017/05/kein-geld-regelbrecher-politische-bedingungen-eu-strukturfonds-ungarn-polen.html.

Araucaria. Revista Iberoamericana de Filosofí, Política, Humanidades y Relaciones Internacionales, año $22, \mathrm{n}^{\circ} 45$. Tercer cuatrimestre de 2020. Pp. 185-205. ISSN 1575-6823 e-ISSN 2340-2199 https://dx.doi.org/10.12795/araucaria.2020.i45.08 


\subsection{El derecho de la competencia y el monopolio mediático}

Más allá de la condicionalidad del MFP, una de las claves del dominio autoritario de Viktor Orbán -el monopolio que ejerce sobre los medios de comunicación de su país (Lendavi, 2017:114)- podría ser disuelto mediante un mecanismo más sencillo: una aplicación creativa del derecho de la competencia.

Según denuncia Sven Giegold, portavoz europarlamentario del Partido Verde alemán, el $78 \%$ de los medios de comunicación húngaros reciben financiación adjudicada, directa o indirectamente, por Fidesz. En los últimos años, Orbán excluyó dos de sus principales armas, el Consejo mediático nacional y la Comisión nacional de competencia, del ámbito del derecho europeo, y creó el "Central European Press and Media Foundation (KESMA)", una fundación liderada por miembros de Fidesz y la cual controla más de 500 medios de comunicación nacionales ${ }^{44}$ Todo ello, añade Paul Lendvai (2017:115), forma parte de una de las prioridades políticas de Orbán desde su derrota electoral en 2002: ejercer un "control absoluto" sobre los medios de comunicación públicos.

En marzo de 2019, respondiendo a una pregunta parlamentaria, la Comisión Europea descartó, "por el momento", la posibilidad de aplicar el derecho de la competencia para contrarrestar dichas medidas. La creación de agencias nacionales como KEMSA, subrayó la Comisión, es competencia de los estados miembro, y no está cubierta por el reglamento 139/2004 (el reglamento comunitario de concentraciones). Solo se podría intervenir, siempre según la Comisión, si el comportamiento de KEMSA afectase al comercio entre estados miembro, y por lo tanto distorsionase el mercado único ${ }^{45}$.

Este análisis, sin embargo, es insatisfactorio. La creatividad jurídica mostrada por la Comisión a la hora de combatir a las grandes plataformas digitales podría servir igualmente para disolver el monopolio ejercido por Fidesz. Tanto el artículo 101 (prácticas comerciales anticompetitivas) como el 102 TFUE (explotación abusiva de una posición dominante) serían de utilidad, mientras que una aplicación inteligente del artículo 106 (que regula las ayudas estatales) permitiría a la Comisión, concluye Giegold, sancionar los subsidios a medios afines al gobierno.

Una vez más, por lo tanto, la pasividad de las instituciones europeas no puede ser reducida, meramente, a una falta de instrumentos legales: es necesaria una mayor voluntad por parte de las instituciones, y un orden de prioridades políticas que refleje que las violaciones del estado de derecho en múltiples

\footnotetext{
44 Sven Giegold (2 de mayo, 2019). Green study reveals dangerous media concentration in Hungary: 78 percent of news media under Orban's control. Recuperado de https://sven-giegold.de/en/ dangerous-media-concentration-in-hungary-78-percent-under-orbans-control/

${ }_{45}$ Parlamento Europeo (18 de marzo, 2019). Answer given by Ms Gabriel on behalf of the European Commission. Question reference: E-006152/2018. Recuperado de https://www.europarl. europa.eu/doceo/document/E-8-2018-006152-ASW_EN.html.
} 
Estados miembro no son un problema secundario, sino una crisis que cuestiona la propia raison d'être de la Unión Europea.

\section{Conclusión: ¿hacia una reforma institucional?}

Es innegable que el fondo de recuperación ha supuesto un momento histórico para la UE. Sin un régimen que supedite el acceso a los fondos al respeto por los "valores fundamentales" de la UE, sin embargo, dicho hito habrá llegado a través de lo que Kelemen (2020b) denomina un "pacto faustiano".

En gran parte, la reticencia mostrada por las instituciones europeas es estructural. Según Kochenov (2004; 2008), los Criterios de Copenhague no fueron aplicados adecuadamente durante la ampliación de 2004, lo cual devaluó su efectividad como mecanismo de control. Asimismo, las deficiencias del sistema político de la UE - el requisito de unanimidad en el Consejo, el monopolio que la Comisión ejerce sobre los procedimientos de infracción, o el imperfecto sistema de partidos políticos, que protege a los líderes de regímenes autoritarios sin someter a sus aliados a consecuencias electorales- no ha hecho sino dificultar su labor.

No se puede entender el comportamiento de las instituciones europeas, sin embargo, sin tener en cuenta los factores netamente políticos que sustentan el "equilibrio autoritario". Las alianzas entre partidos políticos nacionales, así como el sistema de alianzas que ha tejido el grupo de Visegrado, dentro del propio Consejo Europeo, a lo largo de los últimos años, tienen una gran importancia a la hora de entender tanto la lentitud de la Comisión como la negativa del Consejo de aplicar el artículo 7 TUE. Así mismo, la clara falta de creatividad a la hora de aplicar los distintos instrumentos legales disponibles -la ejecución de los Fondos EIE, el derecho de la competencia, o el reglamento 139/2004 sobre el control de las concentraciones entre empresas- demuestra que el problema no es meramente competencial, sino que existe una clara falta de voluntad política (Halmai, 2018).

La disyuntiva entre profundizar la Unión y cumplir -y hacer cumplirel Estado de derecho es falsa: tanto la Comisión como el Consejo disponen de mecanismos suficientes para romper el presente "equilibrio autoritario", requiriendo el respeto por la separación de poderes o la libertad de expresión, y dejando claro que los innegables beneficios económicos que conlleva la pertenencia a la UE vienen acompañados de obligaciones (Kelemen y Scheppele, 2018; Butler, 2018a). Un fondo de recuperación que excluya este mecanismo no solo mandará un mensaje peligroso: que los artículos 2 y 3 del TUE son negociables, y por lo tanto secundarios, sino que permitirá a algunos Estados 
miembro, concluye la columna Charlemagne en The Economist, "luchar contra la UE de lunes a viernes, y recibir sus subsidios los fines de semana"46.

La Conferencia sobre el Futuro de Europa debe hacer lo imposible para sobreponerse a las reticencias de algunos Estados miembros y adaptar la Unión Europea a un contexto geopolítico menos solidario y más aislacionista, tanto fuera como dentro del propio Consejo. Supeditar el fondo de recuperación al respeto por el estado de derecho, facilitar el uso del procedimiento de infracción y reducir la capacidad de veto de los estados miembros no debilitará a la Unión, como se ha sugerido en repetidas ocasiones, sino que le asegurará un funcionamiento más eficaz, facilitando los acuerdos políticos y protegiendo sus valores fundacionales.

Las propuestas del Parlamento Europeo son un buen comienzo; la pelota está en el tejado del Consejo y de la Comisión.

\footnotetext{
${ }^{46}$ Charlemagne (5 de abril, 2018). The EU is tolerating - and enabling - authoritarian kleptocracy in Hungary. The Economist. Recuperado de https://www.economist.com/europe/2018/04/05/the-euis-tolerating-and-enabling-authoritarian-kleptocracy-in-hungary
} 


\section{Referencias bibliográfica:}

Butler, I. (2018a). Dos propuestas para promover y proteger los valores europeos mediante el Marco financiero plurianual. Civil Liberties Union for Europe. Recuperado de https://drive.google.com/file/d/1QFT9LSOiB ojC9hmIsnuPKim943xkcvK9/view.

Butler, I. (2018b). Two Proposals to promote and protect European values through the Multiannual Financial Framework. Civil Liberties Union for Europe. Recuperado de https://drive.google.com/file/ d/1UG4PIg7tObjUoK9tBKq3IdqCT-eB5iM9/view.

Cianetti, L., Dawson, J., and Hanley, S. (2018). Rethinking "democratic backsliding" in central and Eastern Europe - looking beyond Hungary and Poland. East European Politics, 34(3), 243-256.

Closa, C. (2019). The politics of guarding the Treaties: Commission scrutiny of rule of law compliance, Journal of European Public Policy, 26(5), 696716

Comisión de Venecia (2013). Opinion on the fourth amendment to the fundamental law of Hungary, Opinion 720/2013.

Comisión Europea (2020a). Rule of Law 2020: Country Reports. Recuperado de https://ec.europa.eu/info/sites/info/files/rule_of_law_2020_country_ reports_2_web.pdf.

Comisión Europea (2020b). 2020 Rule of Law Report: Country Chapter on the rule of law in Hungary, SWD(2020) 316 final.

Garai, N. (2018). Challenges faced by the Visegrad Group in the 'European dimension' of cooperation. International Issues \& Slovak Foreign Policy Affairs, 27(1-2), Visegrad Praised and Cursed, 24-42.

Gormley, L.W. (2017). Infringement Proceedings. En The Enforcement of EU Law and Values: Ensuring Member States' Compliance (65-78). Oxford: Oxford University Press.

Hooghe, L., Marks, G., y Wilson, C.J. (2002). Does Left/Right Structure Party Positions on European Integration? Comparative Political Studies, 35(8), 965-989.

Íñiguez, G. (2020a, May 24). Quo vadis, Consilium? Agenda Pública. Recuperado de http://agendapublica.elpais.com/quo-vadis-consilium/.

Íñiguez, G. (2020b, 1 de septiembre). El estado de derecho, el gran ausente en el fondo de recuperación. Agenda Pública. Recuperado de http:// agendapublica.elpais.com/el-estado-de-derecho-el-gran-ausente-en-elfondo-de-recuperacion/.

Jakab, A., y Kochenov, D. (eds.) (2017). The Enforcement of EU Law and Values: Ensuring Member States ' Compliance. Oxford: Oxford University Press. 
Kelemen, D.R. (2020a). The European Union's authoritarian equilibrium, Journal of European Public Policy, 27(3), 481-499.

Kelemen, D.R. (2020b). Europe's Faustian Union. Foreign Policy. Recuperado de https://foreignpolicy.com/2020/07/30/europes-faustian-union/.

Kelemen, D.R., y Scheppele, K.L. (2018). How to Stop Funding Autocracy in the EU. Verfassungsblog. Recuperado de https://verfassungsblog.de/howto-stop-funding-autocracy-in-the-eu/.

Kochenov, D. (2004). Behind the Copenhagen façade. The meaning and structure of the Copenhagen political criterion of democracy and the rule of law. European Integration online Papers (EIoP) 8:10. http://eiop.or.at/ eiop/texte/2004-010a.htm.

Kochenov, D. (2008). EU Enlargement and the Failure of Conditionality: PreAccession Conditionality in the Fields of Democracy and the Rule of Law. Alphen aan den Rijn: Wolters Kluwer Law \& Business.

Lendavi, P. (2017). Orbán: Europe's New Strongman. Londres: Hurst.

Levitsky, S., and Way, L. (2010). Competitive authoritarianism. Cambridge: Cambridge University Press.

Müller, J-W. (2014). Rising to the Challenge of Constitutional Capture. Eurozine. Recuperado de https://www.eurozine.com/rising-to-thechallenge-of-constitutional-capture/.

Müller, J-W. (2015). 'Should the EU protect democracy and the rule of law inside Member States?' European Law Journal, 21(2), 141.

Parlamento Europeo (12 de septiembre, 2018). Resolución del Parlamento Europeo sobre una propuesta en la que solicita al Consejo que, de conformidad con el artículo 7, apartado 1, del Tratado de la Unión Europea, constate la existencia de un riesgo claro de violación grave por parte de Hungría de los valores en los que se fundamenta la Unión, 2017/2131(INL). Recuperado de https:/www.europarl.europa.eu/doceo/ document/TA-8-2018-0340_ES.html.

Pech, L. and Scheppele, K.L. (2017). Illiberalism Within: Rule of Law Backsliding in the EU. Cambridge Yearbook of European Legal Studies, 19, 3-47.

Rodríguez Aguilera de Prat, C. (28 de julio, 2020). ¿Puede la Unión Europea superar el estado confederal? Agenda Pública. Recuperado de http:// agendapublica.elpais.com/puede-la-union-europea-superar-el-estadioconfederal/.

Scheppele, K.L. (2016). EU can still block Hungary's veto on Polish sanctions. POLITICO. Retrieved from https://www.politico.eu/article/eu-can-stillblock-hungarys-orban-veto-on-polish-pis-sanctions/. 
Sierakowski, S. (29 de julio, 2020). Europe Bails out its Populists. Project Syndicate. Recuperado de https://www.project-syndicate.org/commentary/ eu-recovery-fund-weakens-rule-of-law-poland-hungary-by-slawomirsierakowski-2020-07?barrier=accesspaylog

Statistisches Bundesamt (2020). Foreign Trade. Ranking of Germany's trading partners in foreign trade (preliminary results). Recuperado de https:// www.destatis.de/EN/Themes/Economy/Foreign-Trade/Tables/order-rankgermany-trading-partners.pdf?_blob=publicationFile.

Szente, Z. (2017). Challenging the Basic Values - Problems in the Rule of Law in Hungary and the Failure of the EU to Tackle Them. En The Enforcement of EU Law and Values: Ensuring Member States' Compliance (456-475). Oxford: Oxford University Press.

Vassileva, R. (2020, September 12). On Coins, Parallel Universes and the Cooperation and Verification Mechanism. Verfassungsblog. Recuperado de https://verfassungsblog.de/on-coins-parallel-universes-and-thecooperation-and-verification-mechanism/.

Way, L y Levitsky, S. (4 de enero, 2019). How autocrats can rig the game and damage democracy. Washington Post. Recuperado de https://www. washingtonpost.com/news/monkey-cage/wp/2019/01/04/how-do-youknow-when-a-democracy-has-slipped-over-into-autocracy/ 
